\title{
AN UNUSUAL LOCATION OF COMPLEX ODONTOME- A DIAGNOSTIC DILEMMA
}

\author{
Saravana Selvam $S^{1}$, C. S. Chandana ${ }^{2}$, M. Indumathi', Pavithra ${ }^{4}$, C. S. Krishnan ${ }^{5}$
}

${ }^{1}$ Reader, Department of Oral Medicine and Radiology, Thai Moogambigai Dental College and Hospital, Chennai, Tamilnadu, India. ${ }^{2}$ Reader, Department of Conservative Dentistry and Endodontics, Thai Moogambigai Dental College and Hospital, Chennai, Tamilnadu, India.

${ }^{3}$ Senior Lecturer, Department of Conservative Dentistry and Endodontics, Thai Moogambigai Dental College and Hospital, Chennai, Tamilnadu, India.

${ }^{4}$ Senior Lecturer, Department of Oral Pathology, Thai Moogambigai Dental College and Hospital, Chennai, Tamilnadu, India. 5 Professor, Department of Periodontology, Madha Dental College and Hospital Tamilnadu, India.

HOW TO CITE THIS ARTICLE: Selvam SS, Chandana CS, Indumathi M, et al. An unusual location of complex odontome- a diagnostic dilemma. J. Evolution Med. Dent. Sci. 2019;8(02):160-162, DOI: 10.14260/jemds/2019/35

\section{PRESENTATION OF CASE}

A male patient aged 35 years came to OPD with a chief illness of broken tooth in relation to left upper front tooth region for past 1 year. Patient was apparently normal before 1 year; then he developed dental caries in relation to left maxillary anterior region because he didn't take much care which resulted in erosion of the entire crown portion of the teeth. Medical history, family history were all examined and found to be normal.

\section{PATHOLOGICAL DISCUSSION}

To find out any periapical infection of the tooth a radiographic investigation of IOPA was planned and taken which revealed loss of crown structure in relation to left maxillary anterior suggestive of root stump, and an additional finding of a welldefined radiopacity mass was evident in relation to periapical aspect of left maxillary anterior region (Figure 1). This was purely an accidental finding and to find out the extent of the structure other imaging modalities like OPG and Occlusal was taken in which OPG revealed well defined agglomerated radiopaque mass measuring approximately about $1 \times 1 \mathrm{~cm}$ in dimension in relation to the periapical aspect of left maxillary anterior region (Figure 2). Occlusal Radiograph discovered a well definite radiopaque mass in relation toward the periapical aspect of left maxillary anterior region near to the midline of nasal septum with irregular structures (Figure 3).

These imaging modalities helped us to locate the structure but the exact extend and its structure was not clear. Hence we went on with advanced imaging modalities which revealed the exact location and extent of the structure. Then with the informed consent from the patient biopsy was performed under Local Anaesthesia (Figure 4). The odontome was removed surgically (Figure 5) and measured using metal caliber (Figure 6). Continuous Interrupted Sutures were placed following the procedure. (Figure7). The tissue was sent to histopathological evaluation which exposed the presence of enamel dentin pulp and cementum suggestive of a Complex odontoma. (Figure 8).

'Financial or Other Competing Interest': None. Submission 05-12-2018, Peer Review 01-01-2019,

Acceptance 07-01-2019, Published 14-01-2019.

Corresponding Author:

Dr. Saravana Selvam $S$,

Reader, Department Oral Medicine and Radiology,

Thai Moogambigai Dental College and Hospital,

Chennai, Tamilnadu, India.

E-mail: drsaravana2512@gmail.com

DOI: $10.14260 /$ jemds $/ 2019 / 35$

(c) $($ ) $\odot$
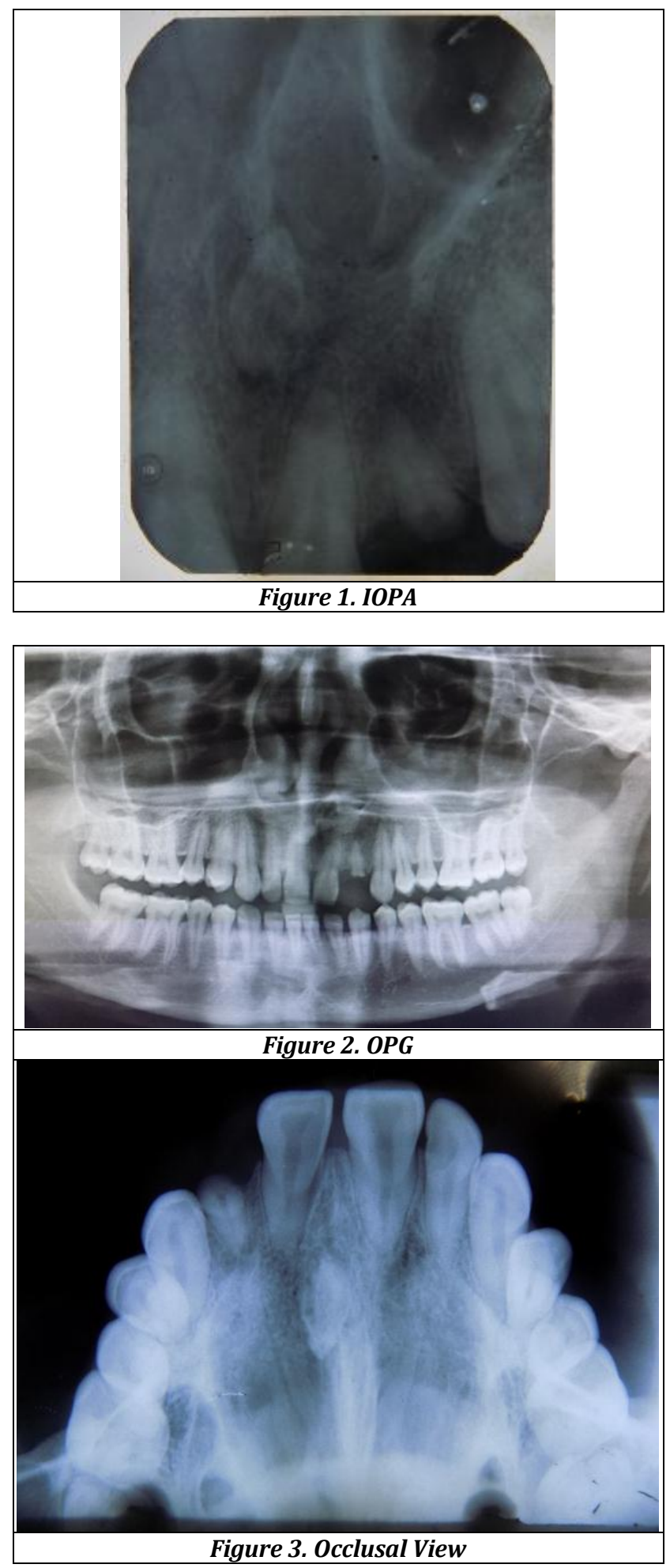

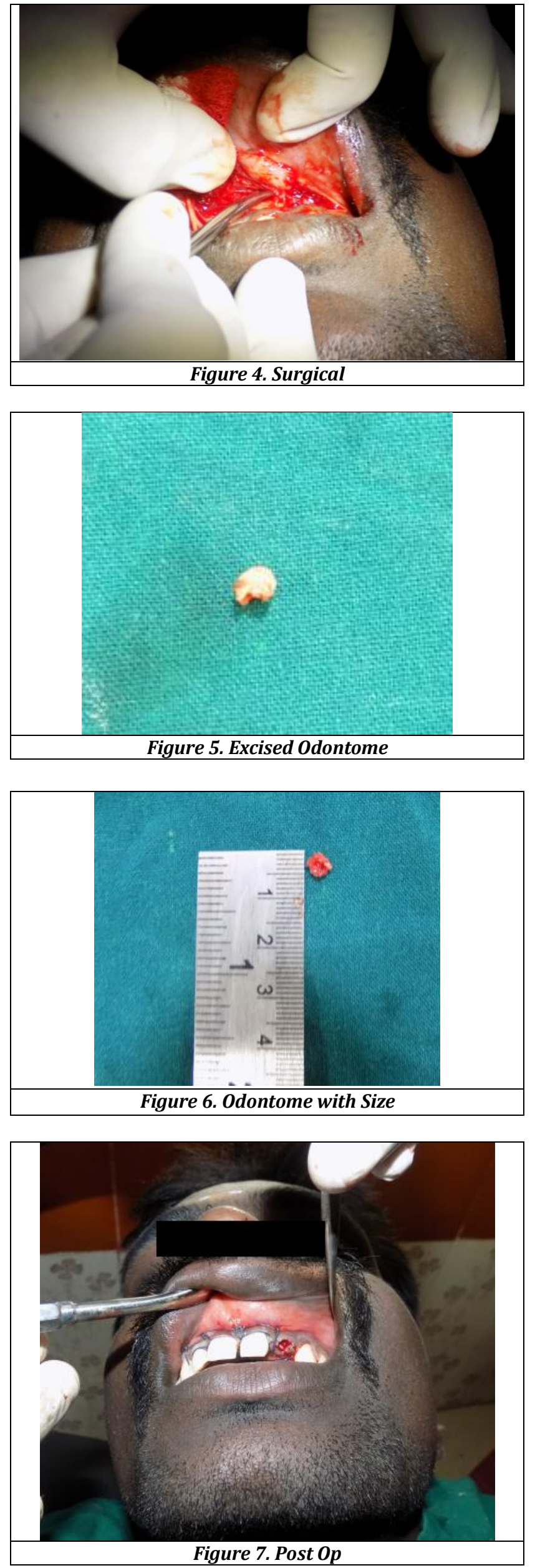

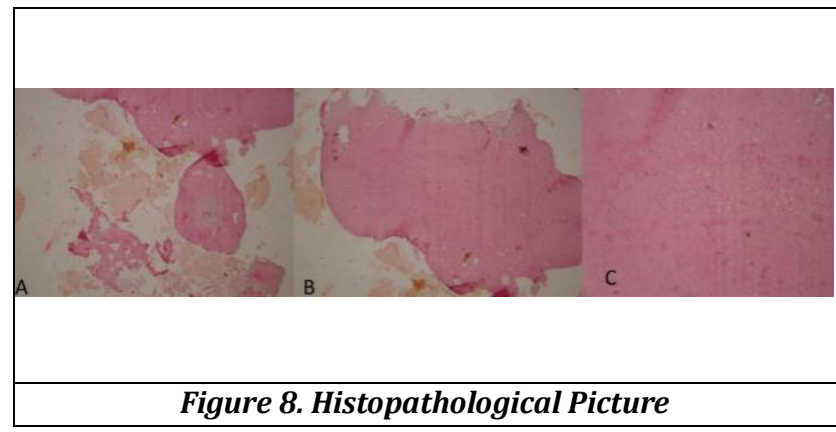

\section{CLINICAL DIAGNOSIS}

Hamartomatous lesion of Odontogenic origin where the epithelial and mesenchymal cells demonstrate absolute demarcation and every part of dental tissue represented is a Complex Odontoma 1. The word Odontoma was termed by Paul Broca in the year 1867 and he demonstrated tumour as created by overgrowth (or) fleeting of entire dental tissue. ${ }^{2}$

It was classified into Complex and Compound Odontoma by World Health Organization (WHO) ${ }^{3}$ where complex stands for irregularity in dental tissue formed and compound stands for regularly formed tissue. 4

\section{DIFFERENTIAL DIAGNOSIS}

Anterior part of Maxilla is the commonest site for Compound Odontoma and posterior part of the mandible is the common location of Complex odontoma. ${ }^{5}$ It can occur at any age mostly 20-30 years of life, there is no gender predilection and soft tissues which grow in a hastily manner. The occurrence of odontoma is the second most frequent odontogenic tumour, even though the complex odontome in an anterior maxillary region are considered to be rare and difficult in diagnosis and management of such an asymptomatic lesion is challenging in practice. ${ }^{6}$

Though the cause of Complex Odontoma is not obviously identified, quite a few hypotheses have been proposed which compromise familial history, trauma and gene mutation. ${ }^{7}$ Mostly seen in males and in posterior part of mandible.8,9 Complex odontomas are generally asymptomatic in nature and found accidentally. ${ }^{8}$

It was suggested that complex odontomas are inherited from a mutant gene or interference, possibly postnatal, with the genetic control of tooth development.10

In majority of kids, these tumours are connected with tooth eruption trouble, such as belated eruption of the deciduous and permanent teeth or overly retained deciduous teeth. $49 \%$ of odontoma diagnosed as a result of belated eruption, $28 \%$ the retention of primary teeth, $20 \%$ are accidentally found on radiographs, and $3 \%$ the swelling of the jaws. ${ }^{11}$

The radiological demonstration of complex odontomas depends on their progressive stage and degree of mineralization. The primary stage is characterized by radiolucency due to a deficiency of calcification. Half-done calcification is observed in the intermediate stage, while in the third stage, the lesion typically appears radiopaque with shapeless masses of the dental hard tissue surrounded by a thin radiolucent zone corresponding to the connective capsule histopathologically. ${ }^{12}$ 
Histopathologically the odontoma is not an investigative dilemma. It is composed of enamel, dentin, cementum, and pulpal tissue. However, mature enamel is lost during the decalcification processing and absent on haematoxylin and eosin stained slides. The compound odontoma recapitulates the association of a normal tooth but have less calcified pulp, primary dentine and enamel than the normal primary dental cement structure and as bone trabeculate between the denticles. The complex odontoma appears as a jumbled mass of hard odontogenic tissues and are characterized by primary dentine, normal or less calcified enamel, immature cementum and a capsule. ${ }^{13,14}$

Complex odontoma generally overlies impacted teeth but in our case there was no impacted tooth and peg lateral was appreciated in relation to left maxillary anterior regions. Complex odontoma commonly lies in right side of maxilla but in this case it was present very near to the midline which was mimicking mesiodens but histopathological investigation ruled it out.

It can be thought of an unknown agglomerated mass like structure the machinery of agglomerated mass eruption appears to be dissimilar from tooth eruption because of deficiency of periodontal ligament and root. Although there is no root formation in agglomerated mass its growing size may lead to the sequestration of overlying bone and hence outcome in occlusal movement or eruption. ${ }^{7}$ In our case all the structures of the teeth were present which ruled out the possible diagnosis of agglomerated mass.

Hamartomousal configuration commonly occurs in the jaw bones. They hardly ever erupt in the oral cavity and are generally associated with an impacted tooth. The reported case is a curious presentation of a complex odontoma which occurred near to midline which is very unusual and also complex odontoma occurring in the anterior part of the maxilla is quite rare. Any radio opacity occurring near to the midline will be mostly interpreted as a mesiodens but other possible diagnosis of complex, compound odontoma and any agglomerated mass must be taken into consideration. As we all know histological diagnosis is the gold standard, but by providing multiple differential diagnoses to a pathologist it makes him to arrive at an accurate diagnosis easily where misdiagnosis will be out of the question. To provide multiple differential diagnoses better understanding and current updates in subject is necessary.

As health care professionals we need to arrive at an accurate diagnosis within short duration for early treatment planning. Knowing the right diagnosis helps in aiding to appropriate treatment planning and the resultant outcome will do wonders to the patient if the clinician is unable to arrive at a diagnosis then seeking an opinion from specific specialist will help in aiding the treatment planning. Clinicians with better knowledge of the radiographic anatomy will be very helpful in arriving at an accurate diagnosis and pinpoint treatment planning. Hence clinicians must be aware of the various radiographic complex structures in the head and neck region so as to eliminate the other possible reasons so correct diagnosis is achieved. Hence knowledge of these complex structures must be emphasized in undergraduate students and separate interest must be given to them in making them identify these lesions so as when they become clinicians it will be of greatest help for them in identifying these lesions.

\section{FINAL DIAGNOSIS}

Complex Odontoma in the Anterior Part of Maxilla.

\section{REFERENCES}

[1] Reichart P, Philipsen HP. Odontogenic tumours and allied lesions. London: Quintessence Publishing 2004: p. 141-7.

[2] Batra P, Gupta S, Rajan K, et al. Odontomes-diagnosis and treatment: a 4 case report. J Pierre Fauchard Acad 2003;19:73-6.

[3] Bordini J Jr, Contar CM, Sarot JR, et al. Multiple compound odontomas in the jaw: case report and analysis of the literature. J Oral Maxillofac Surg 2008;66(12):2617-20.

[4] Shafer WG, Hine MK, Levy BM. Text book of Oral Pathology. $4^{\text {th }}$ edn. Philadelphia: WB Saunders Company 1993: p. 308-12.

[5] Neville BW, Damm DD, Allen CM, et al. Oral and maxillofacial pathology. Philadelphia: Saunders 1995: p. 531-33.

[6] Nagaraj K, Upadhyay M, Yadav S. Impacted maxillary central incisor, canine and second molar with 2 supernumerary Teeth and an odontoma. Am J Orthod Dentofac Orthop 2009;135(3):390-9.

[7] Vengal M, Arora H, Ghosh S, et al. Large erupting complex odontoma: a case report. J Can Dent Assoc 2007;73(2):169-73.

[8] Dua N, Kapila R, Trivedi A, et al. An unusual case of erupted composite complex odontoma. J Dent Sci Res 2011;2(2):1-5.

[9] Serra-Serra G, Berini-Aytes L, Gay-Escoda C. Erupted odontomas: a report of three cases and review of literature. Med Oral Patol Oral Cir Bucal 2009;14(6):E299-303.

[10] Hitchin AD. The aetiology of the calcified composite odontomes. Br Dent J 1971;130(11):475-82.

[11] Tomizawa M, Otsuka Y, Noda T. Clinical observations of odontomas in Japanese children: 39 cases including one recurrent case. Int J Paediatr Dent 2005;15(1):37-43.

[12] Philipsen HP, Reichart PA. Classification of odontogenic tumours. A historical review. J Oral Pathol Med 2006;35(9):525-9.

[13] Nelson BL, Thompson LD. Compound odontoma. Head and Neck Pathol 2010;4(4):290-1.

[14] Diego VJ, Pablo GC, Eduardo CE. Odontoma located within the inferior alveolar nerve: radiographic diagnosis and surgical management of a clinical case. Rev Esp Cir Oral Maxilofac 2009;31(4):264-9. 